\title{
Reliable Battery-Aware Cooperative Multicasting for MBS WiMAX Traffic
}

\author{
Sara Moftah Elrabiei ${ }^{1}$, Moohamed Hadi Habaebi ${ }^{1,2}$ \\ ${ }^{1}$ Department of Computer Engineering, University of Tripoli (Formally Known as Alfateh), Tripoli, Libya \\ ${ }^{2}$ Department of Electrical and Computer Engineering, International Islamic University Malaysia, Kuala Lumpur, Malaysia \\ E-mail: sararabei@hotmail.com,habaebi@ieee.org \\ Received August 22, 2011; revised September 14, 2011; accepted September 26, 2011
}

\begin{abstract}
Recently, relay agents selection schemes were introduced to support downlink multicasting in WiMAX single frequency networks. Such schemes were devised to work cooperatively in order to facilitate reliable Multicast Broadcast Multimedia Services (MBMS) traffic delivery over wireless channels without any consideration to mobile relay agents' battery energy levels. In this paper, we introduce a battery-aware balancing algorithm to operate in conjunction with these relay agents selection schemes proposed in the open literature. A simulation model, used to present the effect of "before" and "after" the battery-awareness selection criterion, highlighted the benefit of using such algorithms in prolonging network lifetime with emphasis on reliable delivery.
\end{abstract}

Keywords: Reliability, Multicasting, WiMAX, MBMS, Battery

\section{Introduction}

Cooperative communication, has gained momentum nowadays, due to its effectiveness and importance in assisting the communication among wireless subscribers, and its ability to enhance capacity of the mobile wireless broadband through multi-hop forward capability. Different users can act as cooperative partners or relay agents $(R A s)$ to assist each other in information transmission, to combat fading in wireless networks, leading to improvement in overall system performance [1-6]. Power consumption is a significant issue and key performance indicator in such a cooperative mobile environment, because of the limited battery power available for mobile termnals that constrains the continuous operation time of wireless devices [7]. Furthermore, multimedia mobile application software nowadays is more processor intensive, and most of the WiMAX MBMS applications last for long durations of time. Therefore, a critical challenge is how to make efficient use of energy, and to extend the battery lifetime for those cooperative relays without jeopardizing their QoS such as throughput and system reliability. In [5], three different re-multicasting schemes were proposed and studied, for IPTV traffic, in conjunction with other schemes available in the open literature. All pro- posed schemes divide the downlink frame into 2-phases (e.g., the first phase is used to receive multicast data from the base station $(B S)$ and the second phase is used to re-multicast it to neighboring mobile subscriber stations (SSs)) but they differ in how to select the $R A$ s to perform such process. Furthermore, none of the selection schemes considered the effect of battery drainage on selected $R A$ s throughout the re-multicasting duration. The contribution of this paper is two-folds. At first, we study the effect of using such re-multicasting schemes without any consideration to the $S S$ battery state and highlight the effect of the selection process on average node energy consumption levels in the network. Secondly, we propose a selection criteria energy level balancing algorithm and implement it into the three different $R A$ s selection schemes and evaluate its effect on system reliability. The rest of this paper is organized as follows. Section 2 introduces the effect of battery drainage of $R A \mathrm{~s}$ in battery unaware network using our MATLAB built simulator. Section 3 discusses the battery aware $R A$ selection optimization schemes. Section 4 introduces the simulation model used. Section 5 evaluates the performance of the proposed schemes and discusses the simulation results, and Section 6 concludes the paper. 


\section{Battery Drain of RAs in Battery Unaware Network}

To achieve a reliable downlink transmission for MBMS traffic and to extend the coverage outside the transmission area, three energy efficient re-multicasting techniques were proposed in [5] for properly selecting $R A \mathrm{~s}$ in a two phase cooperative transmission model. At phase I, the $B S$ multicasts data to all $S S$ s at high transmission rate $R_{1}$, where only subscribers in a good channel state $\left(S S_{G C S}\right)$, e.g., as defined by their CINR threshold, can successfully receive the data, and the remaining group of subscribers in a bad channel state $\left(S S \mathrm{~s}_{B C S}\right)$ fail to receive the data. $B S$ preselects some of $S S_{G C S}$ to be $R A$ s using one of the selection algorithms in Elrabiei et al. [5]. Upon receiving signals from $B S$, each $R A$ decodes the received signals and then forwards them to $S S \mathrm{~s}_{B C S}$ at a proper rate $R_{2}$ in phase II. By exploiting the channel state information (CSI) and the location based service (LBS), a Nearest-Neighbor Discovery Protocol (NNP), and two other optimized versions of it, based on $R A$ 's transmission range and instantaneous CSI, were simulated and studied in the context of WiMAX single frequency networks. These protocols are described briefly below as follow:

\subsection{NNP RA Selection Scheme}

The proposed relay agent selection protocol is based on geographical positioning of users and on the instantaneous channel condition of the SSs. Since we have assumed that BS knows the location of each SS, the assigned RA is chosen to be the nearest located $\mathrm{SS}_{\mathrm{GCS}}$ (neighbor) to the $S S_{B C S}$ of interest, independently of other $S S \mathrm{~s}_{B C S}$. The NNP can be executed periodically according to the mobility of the users and how often they change their locations.

\subsection{Transmission Radius RA Selection Scheme}

The proposed NNP selects a RA for each $S S_{B C S}$ independently of other RAs selection, by finding the nearest $S S_{G C S}$ to the $S S_{B C S}$ from the same MGroup. In worst case scenario, there is independent RA for each $S S_{B C S}$, (i.e., number of RAs equal number of $\mathrm{SSs}_{\mathrm{BCS}}$ ). Therefore, to reduce the number of RAs selected, a scheme optimization is proposed. If there are two or more $S S \mathrm{~s}_{B C S}$, which are in close proximity to each other, the BS can select one RA for all of them if they are located within the RA's transmission radius. In other words, BS chooses the RA with Transmission Radius $x=\arg \min \sum d i$ to the $S S \mathrm{~s}_{B C S}$. For instance, if we consider $\mathrm{d}_{\mathrm{a} 1}, \mathrm{~d}_{\mathrm{a} 2}$, are the distances between $\mathrm{RA}_{\mathrm{a}}$ and $S S 1_{B C S}, S S 2_{B C S}$ respectively, and $\mathrm{d}_{\mathrm{b} 1}, \mathrm{~d}_{\mathrm{b} 2}$, are the distances between $R A_{\mathrm{b}}$ and $S S 1_{B C S \text {, }}$
$\mathrm{SS} 2_{\mathrm{BCS}}$ respectively, then we select the RA which has: $\min \left(d_{a 1}+d_{a 2}, d_{b 1}+d_{b 2}\right)$. The objective of the optimization is to minimize the number of RAs as much as possible, by selecting the $\mathrm{SSS}_{\mathrm{GCS}}$ that can support re-mul- ticasting to the largest number of $\mathrm{SSs}_{\mathrm{BCS}}$ within its coverage transmission range, and not necessary be the nearest $\mathrm{SS}_{\mathrm{GCS}}$ neighbor to each $\mathrm{SS}_{\mathrm{BCS}}$. The template is used to format your paper and style the text. All margins, column widths, line spaces, and text fonts are prescribed; please do not alter them. You may note peculiarities. For example, the head margin in this template measures proportionately more than is customary. This measurement and others are deliberate, using specifications that anticipate your paper as one part of the entire journals, and not as an independent document. Please do not revise any of the current designations.

\subsection{SS-SS Inter-Link CSI RA Selection Algrithm}

The proposed Transmission Radius $R A$ selection algorithm proposed in 2.2 section above reduces further the number of RAs involved in the re-multicasting process but at the expense of algorithm reliability. This is because some of the SSs previously enjoying their own nearby RA are now located at the edge of transmission range of the new RA. Adding that to the varying channel state, it would result into less reliable delivery. In order to compensate for that and to further reduce the number of RAs involved, each $S S$ in the cell is assumed to report also its instantaneous channel quality between itself and its neighboring SSs (i.e., SS-SS inter-link CSI) that can overhear in the cell. Such SS-SS inter-link CSI aggregation can help the $B S$ to select the proper $S S \mathrm{~s}_{G C S}$, that have maximum number of $S S \mathrm{~s}_{B C S}$ within in its transmission range and have good inter-link CSI, to support the re-multicasting. Such technique requires further modifycations to the Wimax Channel Quality Indicator Channel (CQICH) but the implementation is out of the scope of this paper.

The $R A \mathrm{~s}$ are selected so that channel condition between them and all $S S \mathrm{~s}_{B C S}$ has to be in a good state. Using SS-SS instantaneous Inter-link CSI, $R_{2}$ in phase II is determined based on computing the sum of the instantaneous received power from all corresponding $R A \mathrm{~s}$ using the large-scale and small-scale fading channel model, by selecting the Adaptive Modulation and Coding (AMC) level that support the worst CSI between $S S \mathrm{~s}_{B C S}$ and the $R A \mathrm{~s}$. Further details can be found in [5].

The above presented schemes were found to considerably reduce the amount of energy consumed [5], providing a lower cost coverage solution with no dereliction in achieved throughput for all multicast group members. In this study, we have assumed throughout our proposed 
work that in each frame, the $B S$ selects the proper $R A \mathrm{~s}$ for re-multicasting depending on one of the selection techniques discussed above and that the $R A$ 's battery capacity reduces due to re-multicasting process only. But no prior formulation was made to estimate the energy consumption levels or to predict the network energy map. There have been several attempts to address the above issue mentioned especially in the context of wireless sensor networks, where the main source of energy is batteries. Nevertheless, tackling the process of estimating the energy levels at all $S S$ s in the network required in a quantitative closed formula is, somewhat, cumbersome. However, we decided to use qualitative simulation to highlight the effect of our proposed re-multicasting techniques or energy consumption levels. The notations used throughout the paper are listed in Table 1.

\section{Proposed Battery-Aware RA Selection Scheme}

The power consumption and the duration of how long the $S S$ should work as a $R A$ can be adjusted dynamically by the $B S$. The accurate knowledge of the available energy levels in each $S S$ in the network (i.e., energy map of the whole network) is important information for the $B S$ to make its selection. This can be implemented either by allowing the $S S$ to report its battery energy level as extra information sent to the $B S$, or estimated by default as the $B S$ runs the $R A$ selection algorithms and has a priori knowledge of the $R A$ s selection frequency rate. We have resorted to the first assumption in our simulation study.

All SSs are involved in aggregating the CSI map of the network to the $B S$. Therefore, it is similarly possible to

Table 1. Notations definitions (true unless stated otherwise).

\begin{tabular}{cl}
\hline Notation & \\
\hline$R A$ & Relay agent \\
$S S_{B C S}$ & Subscriber station with bad channel state \\
$S S_{G C S}$ & Subscriber station with good channel state \\
$S S_{i, j}$ & The $j$-th group member in $M G r o u p_{i}$ \\
$R_{i}^{1}$ & The rate of the $B S$ in Phase I for MGroup $_{i}$ \\
$R_{i}^{2}$ & The rate of each cooperative transmitter in Phase II for \\
$T_{i}^{1}$ & MGroup $i$ \\
$T_{i}^{2}$ & The transmission time of Phase I for $M G r o u p_{i}$ \\
$\pi_{i}$ & The transmission time of Phase II for $M G r o u p_{i}$ \\
$E_{i, j}^{1}$ & The received signal power for $S S_{i, j}$ in Phase I \\
$p$ & The power \\
$d_{i}$ & Distance between $S S_{i}$ and $B S$ \\
$b_{n}$ & The lower boundaries of signal to noise ratio (SNR) for \\
$N_{0}$ & MCS level $n$ \\
& The noise power \\
\hline
\end{tabular}

map the network mobile SSs battery draining levels at least in a coarse grained fashion. Selected $R A$ s can be considered based on their CSI, geographic locations in the cell, and their battery draining levels. $S S \mathrm{~s}_{G C S}$ that have low battery levels can be spared from the down-link phase II re-multicasting process until recharged. SSs with stationery position and infinite power supply can act as $R A$ s similarly to stationary $R S s$ proposed in $16 \mathrm{j}$ standard, although they don't have to re-multicast continuously on every frame. Similar schemes are used extensively in routing network traffic over wireless links. Therefore we proposed the following battery aware $R A$ selection algorithm described in the following pseudo-code:

\begin{tabular}{l} 
Pseudo Code for Battery-Aware RA Selection Algorithm \\
\hline Schedule the appropriate MGroup ${ }_{i}$ using algorithm described in [6] \\
Locate all the $S S S_{B C S}$ \\
Locate all the $S S s_{G C S}$ \\
For each frame, collect the battery capacity levels in all $S S s_{G C S}$ \\
Compute the average battery available in all mobile $S S s_{G C S}$ \\
Eliminate all $S S_{G C S}$ that have battery capacity less than the computed \\
average battery capacity (e.g., average battery capacity of the cell is \\
computed by taking the average of battery capacity counter values of \\
all mobile $S S s_{G C S}$ involved in the RA selection process only) from RAs \\
selections \\
Select the RAs from the new set of $S S_{G C S}$ based on one of the proposed \\
RA selection algorithms described in [5] \\
Determine $T_{1}, R_{1}, T_{2}, R_{2}$ described in $[5]$ \\
At phase I: BS transmits with $R_{1}$ at $T_{1}$ \\
At phase II: All RAs retransmit with $R_{2}$ at $T_{2}$ \\
Decrement the capacity counter value of the battery $($ drain_level) for \\
each RA selected based on the formula drain_level = $T_{2} /\left(T_{1}+T_{2}\right)$ \\
\hline
\end{tabular}

Once battery capacity levels of all $S S s_{G C S}$ are aggregated to the BS, the algorithm simply excludes certain $S S s_{G C S}$, which have battery energy levels below the average battery capacity of all mobile $S S s_{G C S}$ in the cell, from the RA's selection process. Such approach relieves these $S S s_{G C S}$ from draining their batteries and averages out the RA selection process, thus, prolonging the network lifetime and, consequently, improving network reliability in general.

Once battery capacity levels of all $S S s_{G C S}$ are aggregated to the BS, the algorithm simply excludes certain $S S s_{G C S}$, which have battery energy levels below the average battery capacity of all mobile $S S s_{G C S}$ in the cell, from the RA's selection process. Such approach relieves these $S S s_{G C S}$ from draining their batteries and averages out the RA selection process, thus, prolonging the network lifetime and, consequently, improving network reliability in general.

We have formulated a general term for the probability 
of any randomly picked $S S_{j}$ becoming a $R A$ in the $M_{\text {Group }_{i}}$ that is updated in the battery-aware network [8], and can be estimated using the following formula:

$$
\begin{gathered}
P_{R A}\left(S S_{i j}\right)=\pi_{i} \cdot P\left(E_{i, j}^{1} \geq b_{n}\right) . \\
P\left(d_{j, S S_{B C S}}=\min \left(d_{1}, d_{2}, \cdots, d_{N_{i-1}}\right)\right) . \\
P(\text { battery power }>\text { threshold })
\end{gathered}
$$

where $P_{R A}\left(S S_{i j}\right)$ is the probability of a $S S_{i}$ to be a $R A$ in MGroup $_{i}, \pi_{i}$ is the Service Probability for MGroup given by [6, Equation (6)], $P\left(E_{i, j}^{1} \geq b_{n} N_{0}\right)$ is the probability that $S S_{j}$ has a good CSI (i.e. $S S_{j}=S S_{G C S}$ ), the term $P\left(d_{j, S S_{B C S}}=\min \left(d_{1}, d_{2}, \cdots, d_{N_{i-1}}\right)\right)$ is the probability that $S S_{j}$ is nearest neighbor to the $S S_{B C S}$, and the term $P$ (battery power $>$ threshold) is probability that the battery capacity at the $S S_{j}$ is above a threshold value set by the $B S$ to ensure the ability of re-multicasting. Further details on the mathematical formulation are presented in [8].

\section{Simulation Model}

We have used in this work a channel model that incorporates both large-scale and small-scale fadings that are represented by propagation path-loss, shadowing, and random Rayleigh fading channel $[9,10]$, and no mutual interference is assumed because the SSs use the orthogonal channel. As for the adaptive modulation and coding, the combinations of modulation and coding set (MCS) levels for the IEEE 802.16 air-interface are shown in Table 3 in the first four columns, where $b_{n}$ represents the lower boundaries of SNR (receiver minimum sensitivity level), and $r_{n}$ is the corresponding discrete peak transmission rate for the state $n$ used in this work for $10 \mathrm{MHz}$ system. State 0 represents the state that no transmission is allowed, which occurs when there is poor channel condition.

We compare the performance for the three systems, before and after battery-awareness situations, using extensive simulations with Matlab. In our simulated system, the network is composed of one $B S$ and a number of $S S$ s which are randomly and uniformly distributed over the coverage area of the $B S$ (i.e., a circle centered at the $B S$ with a radius of $\approx 2.5 \mathrm{~km}$ ). There are different MGroups in the network and each group includes some members which are randomly selected from the whole SSs set. Channel model described in above is applied, and the scheduling scheme for MGroups proposed in [4] is utilized so that the throughput performance and fair scheduling is not jeopardized. The main system simulation parameters are listed in Table 2 and their descriptions are discussed in [5]. We have repeated the simulation several times with different random seeds to calculate the average results.
Table 2. System parameters.

\begin{tabular}{ll}
\hline Number of cells & 1 \\
\hline Carrier frequency \& BW & $2.5 \mathrm{GHz}, 10 \mathrm{MHz}$ \\
Cell radius & $2.56 \mathrm{Km}$ \\
The total number of SSs in the system & 200 \\
The number of $M G r o u p s ~$ & 16 \\
The number of group members in each $M$ Group $N_{i}$ & Varying \\
$B S$ maximum transmission power & $20 \mathrm{~W}$ \\
$S S$ maximum transmission power & $3 \mathrm{~W}$ \\
Noise power & $-99 \mathrm{dBm}$ \\
Path loss exponent $\alpha$ & 4.375 \\
Close-in reference distance $d_{0}$ & $100 \mathrm{~m}$ \\
Lognormal Shadowing & $8.9 \mathrm{~dB} \mathrm{std}$ \\
Coverage ratio $C$ & $50 \%$ \\
Transmitter antenna gain $G_{t}$ & 1 \\
Receiver antenna gain $G_{r}$ & 1 \\
System loss $L$ & 1 \\
DL/UL sub-frame duration & $1.25 \mathrm{~ms} / 1.25 \mathrm{~ms}$ \\
OFDMA symbol duration $\tau$ & $102.9 \mu \mathrm{s}$ \\
Frame duration & $5 \mathrm{~ms}$ \\
Time ratio for multicasting & $25 \%$ \\
\hline
\end{tabular}

Table 3. Modulation and coding schemes for IEEE 802.16.

\begin{tabular}{ccccc}
\hline Level & $\begin{array}{c}\text { Modulation and } \\
\text { coding }\end{array}$ & $\boldsymbol{b}_{\boldsymbol{n}}(\mathbf{d B})$ & $\boldsymbol{r}_{\boldsymbol{n}}$ (Mbps) & $\begin{array}{c}\text { Surface } \\
\text { ratio \% }\end{array}$ \\
\hline 0 & No Tx & 0 & 0 & $27.0782 \%$ \\
1 & BPSK (1/2) & 3 & 2.52 & $19.7459 \%$ \\
2 & QPSK (1/2) & 6 & 5.04 & $12.3036 \%$ \\
3 & QPSK (3/4) & 8.5 & 7.56 & $11.0675 \%$ \\
4 & 16 QAM (1/2) & 11.5 & 10.08 & $9.1849 \%$ \\
5 & 16 QAM (3/4) & 15 & 15.12 & $6.3544 \%$ \\
6 & 64 QAM (2/3) & 18.5 & 20.16 & $3.3007 \%$ \\
7 & 64 QAM (3/4) & 21 & 22.68 & $10.9648 \%$ \\
\hline
\end{tabular}

\section{Performance Evaluation}

\subsection{Battery Energy-Unaware System Performance}

When analyzing our results it appeared that some mobile $S S$ s are selected as $R A$ s more often than their counterparts especially when these $S S$ s were located at places with line of sight connection from the $B S$ and/or have always good channel state. This frequent selection resulted in the depletion of their limited energy sources faster than the average depletion rate of the network.

In Figure 1, the histogram illustrates the number of times a 50 SSs sample were selected as $R A$ s through 600 frames duration time. Increasing the $S S$ density in the cell, resulted in reduction of their severed energy consumption levels but the general trend persisted. Over time, the discharging process drained their batteries and they were automatically eliminated from the network population. Thus, reducing the system reliability in general and bringing the network lifetime to a lesser reach.

In order to investigate the effect of battery levels on 


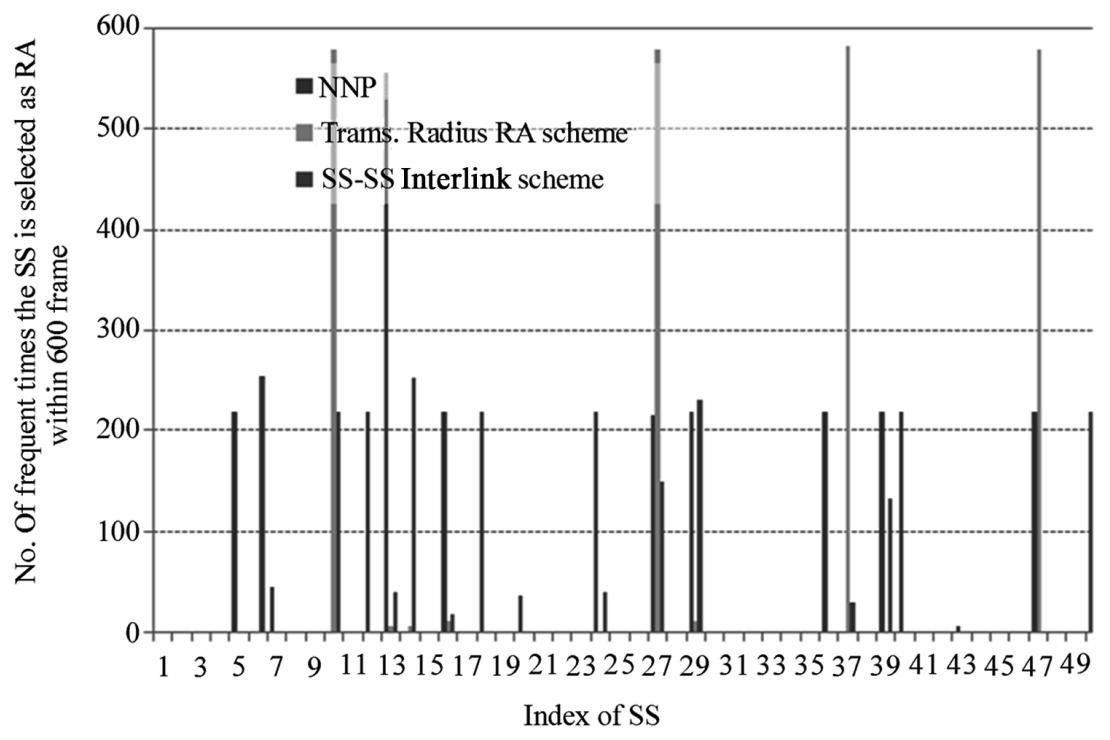

Figure 1. Histogram of number of times the SSs are selected as $R A$ s through the 600 frames.

the network reliability, we have set the number of $S S \mathrm{~s}$ in the cell to $50 \mathrm{SSs}, 10 \%$ of them are stationary with unlimited power supply (e.g., their battery levels are unaffected by the re-multicasting process), while the other $90 \%$ are mobile, slowly cruising around the cell. In order to highlight the performance of the three different schemes introduced in [5], we have isolated the effect of the re-multicasting process on battery state by assuming an initial value of battery state and each re-multicasting frame results in a amount of energy consumed, where, $T_{1}$ and $T_{2}$ are the first and the second phase of the transmission burst $T$ assigned for downlink multicast transmission duration respectively, where $T=T_{1}+T_{2}$. All other energy consumption processes such as UL transmission, DL unicast transmission, processing, etc., were ignored in order to isolate the effect of battery drainage on system performance. We have repeated the simulation several thousand times in order to calculate the average results. The normalized average capacity per mobile $S S_{G C S}$ is calculated for the mobile $S S_{G C S}$ only and not for all $S S \mathrm{~s}$ in the cell.

The effect of the re-multicasting process at each frame on the average battery capacity per mobile $S S_{G C S}$ in the network is plotted in Figure 2. The average battery capacity was normalized relative to the initial battery capacity value of the $S S$ capacity. The results were plotted for the NNP RA scheme, transmission radius $R A$ selection scheme, and SS-SS inter-link CSI RA selection scheme. The average normalized battery capacity is decreasing with increasing the time elapsed. Obviously, decreasing the capacity in each re-multicasting process depends on the duration of the phase II duration $\left(T_{2}\right)$. After 1000 frames the battery capacity per mobile $S S_{G C S}$ reduces to half of the initial value using the optimized selection techniques, whereas the NNP the capacity reduces only $20 \%$ for its initial value. This is due to the fact that our optimized schemes selected $R A \mathrm{~s}$ based on distance and CSI criteria that fit fewer $S S \mathrm{~s}_{G C S}$. These $S S \mathrm{~s}_{G C S}$ were used more often depleting their energy levels quickly in comparison to other $S S$ s bringing in the process the network average battery state to lower levels faster. On the other hand, the NNP algorithm used the simpler selection criterion by allowing more $R A \mathrm{~s}$ in the network to be replaced by other $R A \mathrm{~s}$ more frequently, thus resulting in smoother average battery energy consumption throughout the cell.

The same results were confirmed by the average network reliability performance over time as shown in Figure 3. The general trend is that the reliability decreased with increasing the time elapsed, and overtime, some $R A \mathrm{~s}$ discharged all their battery capacity, hence reducing the number of $R A \mathrm{~s}$ in the network. The optimized schemes are often deeply affected in comparison to the $N N P$, because they have fewer $R A \mathrm{~s}$, when the stationary $R A \mathrm{~s}$ are not affected at all. After 1000 frames, the reliability of the optimized schemes decreased to about $40 \%$, while the NNP reliability reduced only to about $70 \%$.

\subsection{Battery Energy-Aware System Performance}

In this section, the performance of our proposed scheme was calculated in terms of relative power consumption levels and system reliability. Extensive simulations with Matlab were conducted using the implemented battery-aware $R A$ selection schemes. The same simulation set-up was maintained in order to facilitate the "before" 


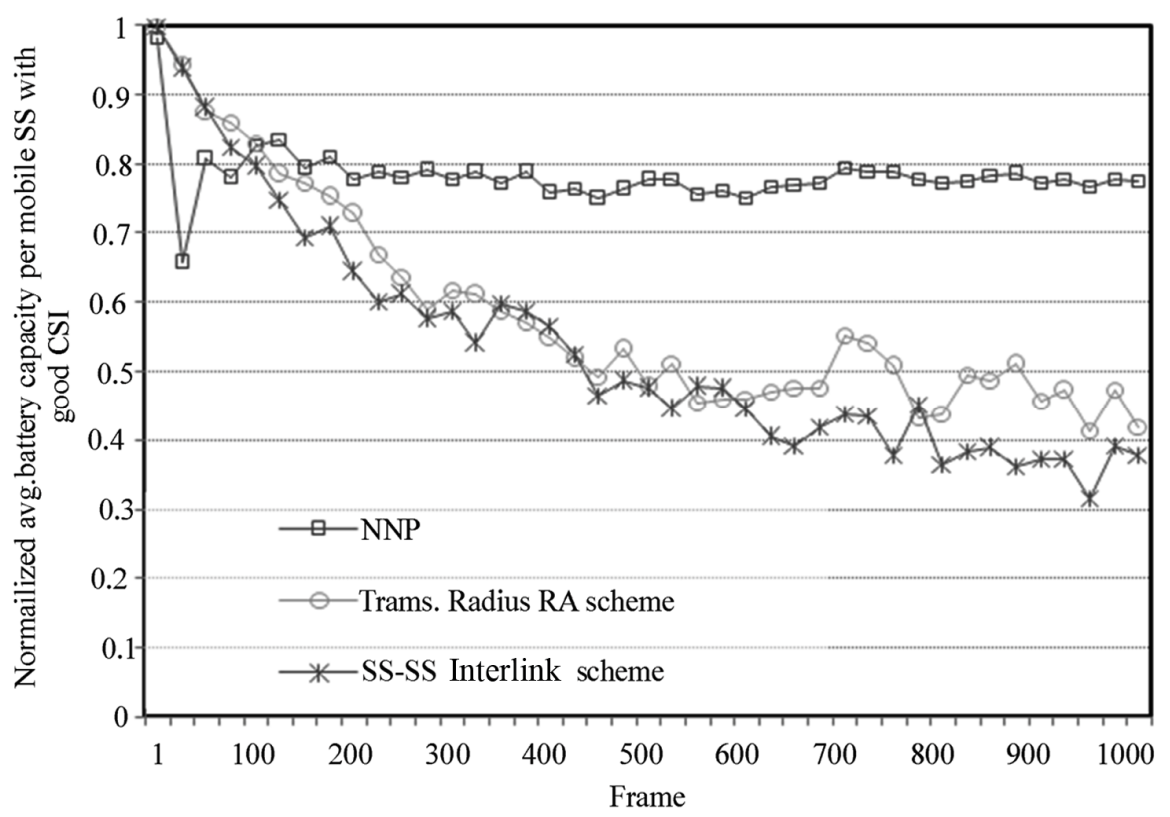

Figure 2. Average normalized battery capacity per mobile $S S_{\mathrm{GCS}}$ versus frame number.

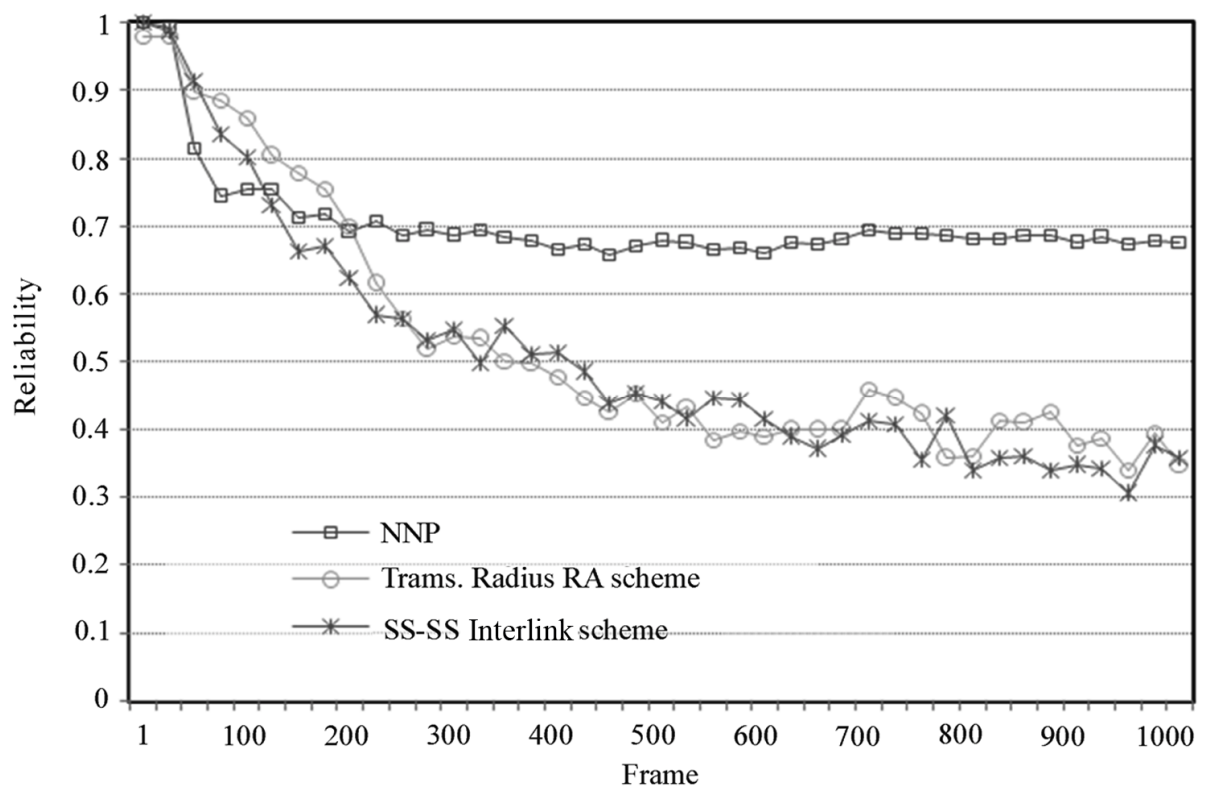

Figure 3. Reliability of the proposed schemes versus frame number.

and "after" comparison. The number of $R A \mathrm{~s}$ is basically dependent on the number of SSs in the cell, the location distribution of $S S \mathrm{~s}_{B C S}$ within the cell and on the wireless channel conditions. The normalized average battery capacity per mobile $S S_{G C S}$ in the network at each frame for all proposed schemes, was computed and plotted in Figure 4. The implementation of the battery-awareness on the three $R A$ selection schemes demonstrated more balanced performance over time achieving almost a steady state.
Similarly, the more balanced performance achieved for all three $R A$ selection schemes is translated into slower degradation in system reliability as illustrated in Figure 5. The battery-aware SS-SS inter-link CSI selection scheme achieved the best performance in comparison to the other two schemes, but the overall results are much better than those shown in Figure 3. This is because the $B S$ always monitors the battery state of the whole network allowing it to fine tune the process of $R A$ selection continuously, hence, prolonging the network life time. 


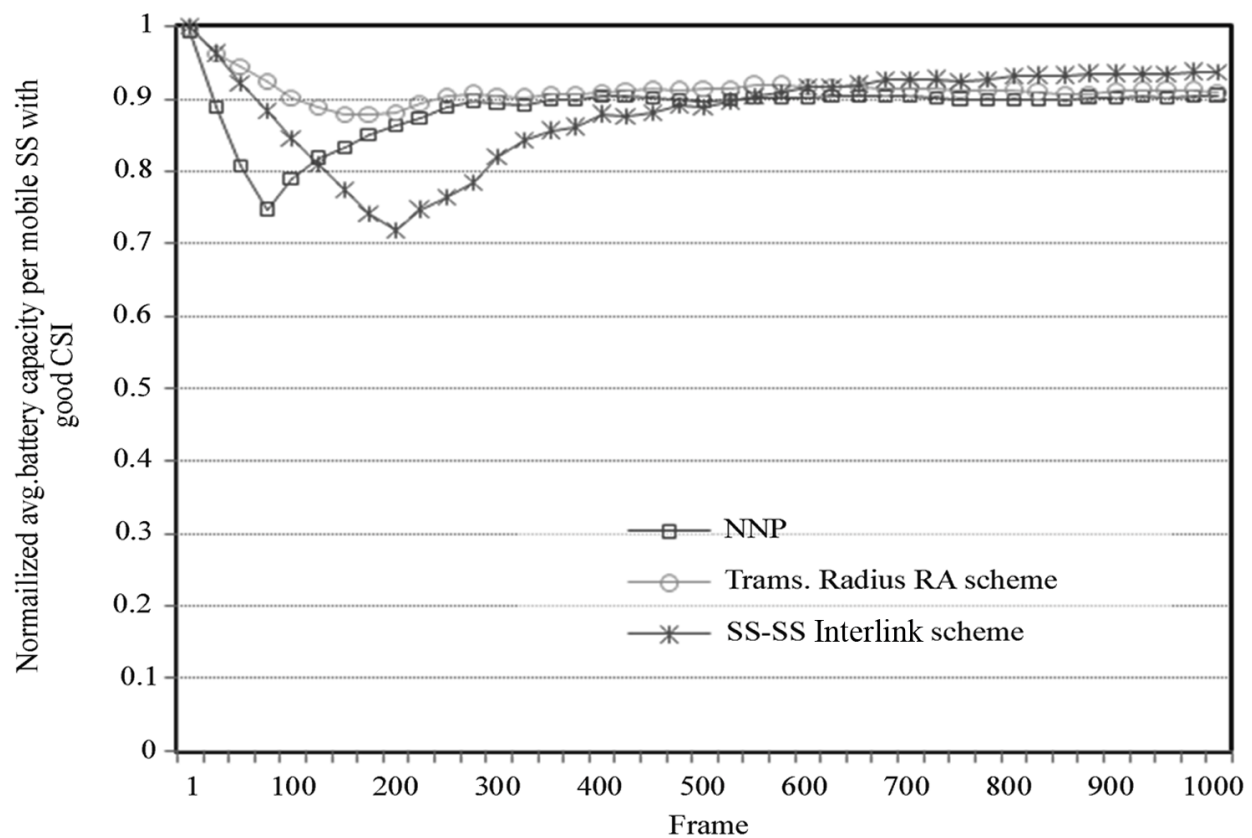

Figure 4. Average normalized battery capacity per mobile $\mathrm{SS}_{G C S}$ versus frame number for the battery aware optimized scheme.

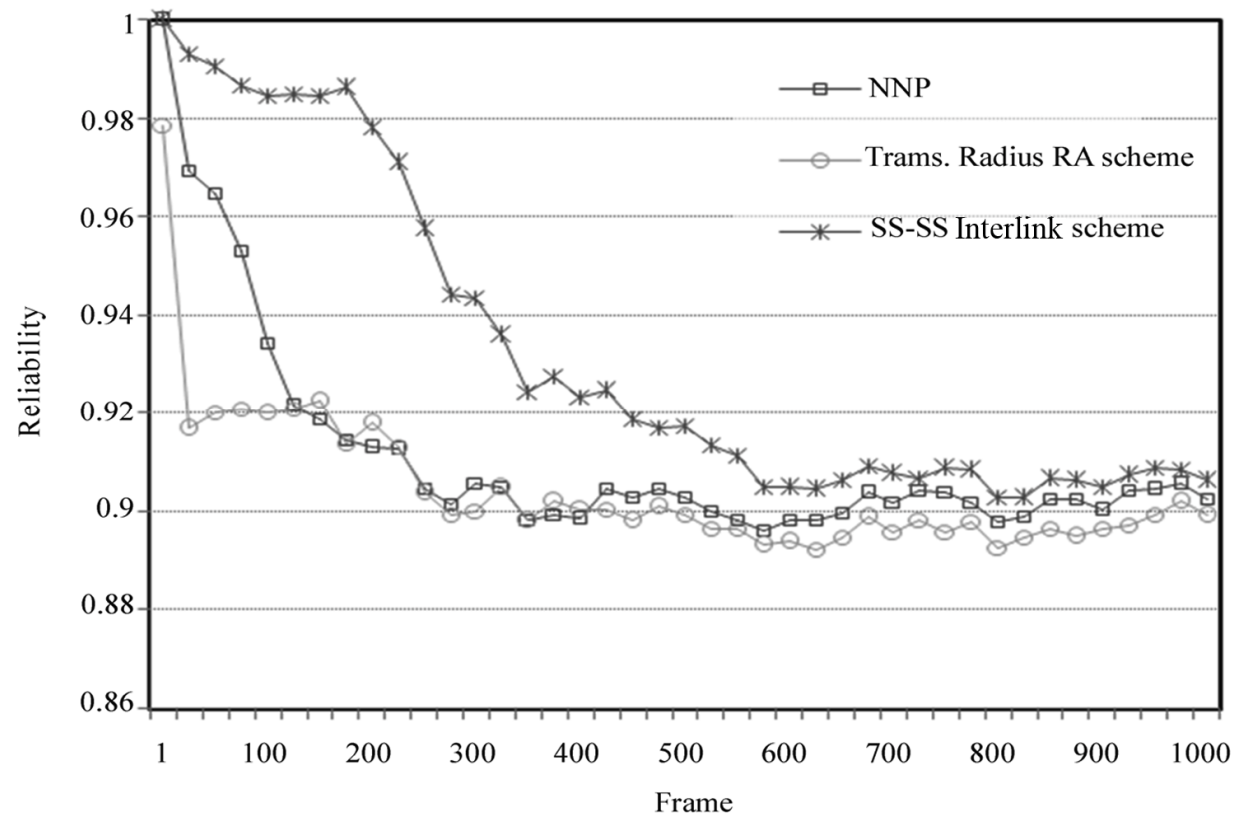

Figure 5. Reliability of the proposed schemes versus frame number for the battery aware optimized scheme.

\section{Conclusions}

The relay agent selection balancing algorithm, introduced in this paper for downlink multicasting traffic over WiMAX channels, demonstrated the effectiveness of considering the battery energy levels of mobile subscriber stations as an additional RA selection criterion when supporting re-multicasting over 2-phased down- link frames. Such added criterion has prolonged the network lifetime by reducing the average node energy consumption levels with emphasis on reliable MBMS traffic delivery. Amongst the algorithms, the battery-aware SS-SS inter-link CSI RA selection scheme achieved the best performance in comparison to the other two $N N P$ and Transmission-Radius RA selection schemes. Nevertheless, the overall results demonstrated more reliable 
delivery over longer periods of time.

\section{References}

[1] Y. Eisenberg and C. Logan, "Cooperative Communi cations for Improved Throughput, Range and Covert ness," Military Communications Conference, IEEE MILCOM 2008, Convention Center, San Diego, 16-19 November 2008, pp. $1-7$.

[2] A. Nosratinia, T. E. Hunter and A. Hedayat, "Cooperative Communication in Wireless Networks," IEEE Communications Magazine, Vol. 42, No. 10, 2004, pp. 74-80. doi:10.1109/MCOM.2004.1341264

[3] P. Liu, Z. Tao, Z. Lin, E. Erkip and S. Panwar, "Cooperative Wireless Communications: A Cross-Layer Approach," IEEE Wireless Communications, Vol. 13, No. 4, 2006, pp. 84-92. doi:10.1109/MWC.2006.1678169

[4] Q. Zhang, J. C. Jia and J. Zhang, "Cooperative Relay to Improve Diversity in Cognitive Radio Resource," IEEE Communications Magazine, Vol. 47, No. 2, 2009, pp. 111117. doi:10.1109/MCOM.2009.4785388

[5] S. M. Elrabiei and M. H. Habaebi, "Energy Efficient Cooperative Multicasting for MBS WiMAX Traffic," 21st Annual International Symposium on Personal, Idoor and Mobile Radio Communications PIMRC'2010, Istanbul, 26-30 September 2010, pp. 1717-1722.

[6] F. Hou, et al., "A Cooperative Multicast Scheduling Scheme for Supporting Multimedia Services over IEEE 802.16 Networks," IEEE Transactions on Wireless Communications, Vol. 8, No. 3, 2009, pp. 1508-1519. doi:10.1109/TWC.2009.080417

[7] A. Ephremides, "Energy Concerns in Wireless Networks," IEEE Wireless Communications, Vol. 9, No. 4, 2002, pp. 48-59. doi:10.1109/MWC.2002.1028877

[8] S. M. Elrabiei and M. H. Habaebi, "Energy Efficient Cooperative Multicasting for MBS WiMAX Traffic," 5th International Symposium on Wireless Pervasive Computing ISWPC'2010, Modena, 5-7 May 2010, pp. 600-605.

[9] J. G. Andrews, A. Ghosh and R. Muhamed, "Fundamentals of WiMAX," Prentice-Hall, Upper Saddle River, 2007.

[10] Theodore S. Rappaport, "Wireless Communications: Prin ciples and Practice," 2nd Edition, Prentice-Hall, Upper Saddle River, 2002. 